\author{
MITSUBISHI ELECTRIC RESEARCH LABORATORIES
}

http://www.merl.com

\title{
Stability Metric Based Routing Protocol for Low-Power and Lossy Networks
}

\author{
Yang, X.; Guo, J.; Orlik, P.; Parsons, K.; Ishibashi, K.
}

TR2014-045 June 2014

\begin{abstract}
To design a routing protocol for applications over low-power and lossy networks (LLNs), the IETF ROLL Working Group standardized the IPv6 Routing Protocol for LLNs (RPL), which organizes nodes in a LLN into a tree-like topology called Destination Oriented Directed Acyclic Graph (DODAG). RPL shows good scalability and fast network setup. However, it may suffer from severe unreliability due to the selection of suboptimal routes with low quality links. To optimize the reliability of RPL routes, this paper proposes a stability metric based routing protocol named sRPL for reliable routing and data collection in LLNs. We introduce a new routing metric for RPL called stability index (SI), which exploits stability characteristics of RPL nodes to select more stable routes. In addition, we present a passive and lightweight network layer technique to measure the bi-directional expected transmission count (ETX) for wireless links in LLNs. As a use case of SI, we combine SI metric with ETX metric to make routing decisions. Simulation results show that SRPL can improve packet delivery rate of RPL routing protocol by $20 \%$.
\end{abstract}

\section{IEEE International Conference on Communications (ICC)}

\footnotetext{
This work may not be copied or reproduced in whole or in part for any commercial purpose. Permission to copy in whole or in part without payment of fee is granted for nonprofit educational and research purposes provided that all such whole or partial copies include the following: a notice that such copying is by permission of Mitsubishi Electric Research Laboratories, Inc.; an acknowledgment of the authors and individual contributions to the work; and all applicable portions of the copyright notice. Copying, reproduction, or republishing for any other purpose shall require a license with payment of fee to Mitsubishi Electric Research Laboratories, Inc. All rights reserved.
} 



\section{Stability Metric Based Routing Protocol for Low-Power and Lossy Networks}

\author{
Xin Yang \\ Scalable Software Systems Lab \\ University of Florida \\ Gainesville, FL 32611, USA \\ xin@cise.ufl.edu
}

\author{
Jianlin Guo, Philip Orlik, Kieran Parsons \\ Electronics and Communications \\ Mitsubishi Electric Research Laboratories \\ Cambridge, MA 02139, USA \\ \{guo, porlik, parsons\}@merl.com
}

\author{
Koichi Ishibashi \\ Wireless Modules Development Center \\ Mitsubishi Electric Corporation IT R\&D Center \\ Ofuna, Japan \\ Ishibashi.Koichi@ce.MitsubishiElectric.co.jp
}

\begin{abstract}
To design a routing protocol for applications over low-power and lossy networks (LLNs), the IETF ROLL Working Group standardized the IPv6 Routing Protocol for LLNs (RPL), which organizes nodes in a LLN into a tree-like topology called Destination Oriented Directed Acyclic Graph (DODAG). RPL shows good scalability and fast network setup. However, it may suffer from severe unreliability due to the selection of suboptimal routes with low quality links. To optimize the reliability of RPL routes, this paper proposes a stability metric based routing protocol named SRPL for reliable routing and data collection in LLNs. We introduce a new routing metric for RPL called stability index (SI), which exploits stability characteristics of RPL nodes to select more stable routes. In addition, we present a passive and lightweight network layer technique to measure the bi-directional expected transmission count (ETX) for wireless links in LLNs. As a use case of SI, we combine SI metric with ETX metric to make routing decisions. Simulation results show that SRPL can improve packet delivery rate of RPL routing protocol by $20 \%$.
\end{abstract}

Keywords-Stability based routing; passive stability measurement; lightweight computation; control message monitoring; advanced metering infrastructure; low power and lossy networks

\section{INTRODUCTION}

Low-power and lossy networks (LLNs) are playing more and more important roles for a wide range of applications such as advanced metering infrastructure (AMI), building automation and environment monitoring. Discovering reliable routes in LLNs is challenging since LLNs are typically composed of a large number of nodes with various physical constraints and such constrained nodes communicate using wireless links in a multihop manner. Fluctuations in the wireless communication environments make network topology unstable and subsequently result in unreliable routing and poor reliability.

The IETF ROLL Working Group standardized RPL in RFC6550 [1]. For routing purposes, RPL organizes LLN nodes into tree-like structures named Destination Oriented Directed Acyclic Graph (DODAG). To achieve reliable routing and data collection, RPL allows a node to have multiple parents in the DODAG structure with one preferred parent for data forwarding. In case the preferred parent becomes unavailable, a backup parent can be used to deliver packets towards the destination. Performance of RPL has been evaluated and analyzed by researchers. RPL shows good scalability [2] and fast network setup [3]. However, RPL may suffer from severe unreliability as shown by [2] and [4] and frequent route

This work was done when Xin Yang worked as an intern at Mitsubish Electric Research Laboratories (MERL). changes have negative impact on the performance of network [5]. RPL defines an Objective Function (OF) for guiding nodes to select parents and construct DODAG topology. Several routing metrics, such as hop count and ETX, are specified [6]. However, a metric that depicts stability of nodes is missing.

In this paper, we propose a stability metric based RPL (sRPL) to discover stable routes in LLNs. There are several challenges arising here. First, the routing protocol for LLNs should be lightweight. Wireless communication resources are valuable, particularly in LLNs in which nodes are equipped with highly constrained resources and feature short range communication capacities. High protocol overhead associated with route discovery and maintenance can drain resources quickly and may also interfere with data collection. Second, the routing protocol at network layer should make no specific assessment on the link layers, which could be IEEE802.15.4, low power WiFi or powerline communication. The independence will benefit routing protocol's adaptability in different types of physical networks and applications. Third, the routing protocol should be scalable. LLNs are typically deployed in an extended area to support large-scale applications. As a result, the global information of a large network is hardly available at each individual node, and the routing protocol should leverage the local information to make proper routing decisions.

To overcome these challenges, sRPL adds a few new designs to RPL framework. In RPL, nodes transmit different types of control messages, which indicate various stability characteristics. Therefore, we introduce a new routing metric, stability index (SI), which uses the transmission rate of control messages to describe the stability of DODAGs and nodes. Based on SI, sRPL selects more stable nodes to form more reliable routes. SRPL measures SI at every node passively by monitoring control messages transmitted by neighboring nodes. Thus, no communication overhead is incurred and no other information is needed. SI can be used as a routing metric to build reliable routes. It can also be weaved together with other routing metrics such as ETX and hop count to optimize route selection.

In addition, we propose a passive and lightweight network layer technique to measure the bi-directional ETX for wireless links. Different from the conventional network layer ETX measurement methods that rely on network layer ACK or link layer feedback, we calculate ETX by passively monitoring data packet transmissions only.

The rest of this paper is organized as follows. Section II 
presents related work. Section III describes the stability characteristics of RPL. Detailed design and measuring of SI and ETX metrics are presented in Section IV. Section V gives the extensive evaluation and analysis based on simulations. We conclude our work in Section VI.

\section{RELATED WORK}

In multihop wireless networks, choosing a proper routing metric to discover reliable routes presents challenges to network layer routing protocols such as RPL. A commonly used network layer metric is hop count. However, a short path may be composed of low quality links and does not necessarily provide high reliability. Metrics that reveal real link quality such as the received signal strength are difficult to obtain at the network layer. Therefore, link layer metrics such as ETX attract a lot of attention since they can be estimated at network layer.

The ETX metric was introduced in [7] to find high throughput routes in wireless networks. The proposed method of measuring ETX for wireless links in IEEE 802.11 networks uses broadcast probe techniques, which incur very high communication overhead. Feasibility of applying broadcast-based metrics in wireless networks is discussed in [8], and authors observed the extreme sensitivity of broadcast-based metrics in their test beds. A single TCP transfer can perturb the metric value of $70 \%$ wireless links with more than half of the links increasing their metric values three times. Kim et al. [9] propose a passive network layer method to estimate ETX by monitoring unicast packets. However, a network layer ACK is needed for each successful packet delivery. Network layer ACK incurrs communication overhead. To eliminate the overhead, ETX estimation is moved to the device driver. As a result, the method proposed is not a network layer technique.

As one of metrics for RPL [6], ETX is used in recent RPL studies. [10] uses ETX as the RPL routing metric for the advanced metering infrastructure (AMI) in Smart Grid. The measurement of ETX relies on IEEE 802.11 ACK mechanism. Limitation is that unicast-based data and ACK approach reveal only uni-directional link quality [9]. Authors of [11] discuss the problems of link quality estimation in dense RPL deployments and propose a passive probing scheme. However, the proposed ETX estimation is also uni-directional and may result in an unstable DODAG topology.

Several technologies have been proposed to improve the performance of RPL. [12] proposes an opportunistic RPL variation, which uses the received signal strength and retransmission rate as metrics in RPL to achieve low cost and stable communication in smart grid systems. The proposed technique requires feedback from MAC and PHY layers and therefore is MAC/PHY protocol dependent. [13] introduces a load balanced technique to improve reliability of RPL. Workload is employed in making routing decision. More packets are sent to parents with lighter workload and fewer packets are sent to parents with heavier workload.

In this paper, we introduce a new routing metric, SI, for RPL protocol to discover stable routes in LLNs. We consider LLNs that consist of multiple data sinks with each data sink being the root of a DODAG. Nodes and data sinks form large scale wireless mesh networks in which nodes typically send their data packets to data sinks in a multihop manner. Based on SI, a node first selects the most stable DODAG to join and then selects the most stable neighbors as parents in the selected DODAG. We also propose a passive and lightweight network layer ETX measurement method that calculates the bidirectional link quality and incurs no communication overhead for periodic data collection and very low communication overhead for non-periodic data collection.

\section{StABILITY CHARACTERISTICS OF RPL}

In this section, we provide a brief overview of RPL protocol and explore stability characteristics of RPL.

\section{A. RPL Overview}

RPL is a routing protocol specifically designed for LLNs. It organizes nodes in a LLN as a Directed Acyclic Graph (DAG) and partitions the DAG into one or more DODAGs, one per data sink. To construct DODAG and upward routes, the data sink acts as the root of DODAG and broadcasts DODAG Information Object (DIO) messages to its neighbors. DIO messages contain the information to construct a DODAG. For example, RPLInstanceID, DODAGID and DODAGVersionNumber are enclosed to identify a DODAG version. Rank defines the node's individual position relative to other nodes with respect to the root of the DODAG. Nodes in the proximity of the data sink receive the DIO messages, compute their own ranks if they decide to join the DODAG, transmit new DIO messages containing their own ranks to their neighbors. DIO messages are propagated, and the DODAG topology is constructed in a wavefront manner, until every node joins a DODAG. RPL uses the Destination Advertisement Object (DAO) messages to build downward routes. The DODAG Information Solicitation (DIS) message is to solicit a DIO from neighboring RPL nodes.

\section{B. Stability and Control Messages in RPL}

In RPL, the transmission rate of control messages, DIS, DIO and DAO, imply stability and connectivity of nodes.

- DIS: A DIS transmission indicates a node's intention to discover a new route.

- DIO: A DIO transmission indicates a node's route information change or a solicitation from a neighboring node for discovering new route.

- DAO: A DAO transmission indicates a node's route information change or route information change of another node in the node's sub-DODAG.

To show the relationship between the control message transmission rate and the stability of node, we simulated a wireless network of 1024 nodes with RPL routing protocol by using the NS2 simulator. Each node in the network sends a data packet to the data sink every 5 minutes. We used the radio propagation models of two-ray ground and shadowing to simulate the stable connectivity and the unstable connectivity, respectively. The CDF of the number of control messages transmitted is plotted in Fig. 1. For the 24-hour simulation time, we observed that $80 \%$ of nodes in stable-connectivity network transmit fewer than 100 control messages, but $98 \%$ of nodes in unstable-connectivity network transmit more than 200 


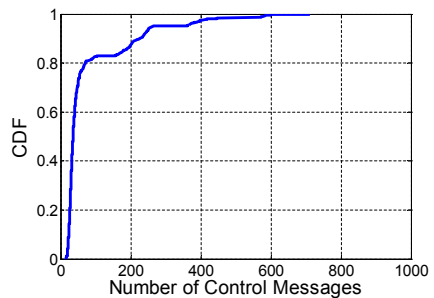

(a) Stable-connectivity network

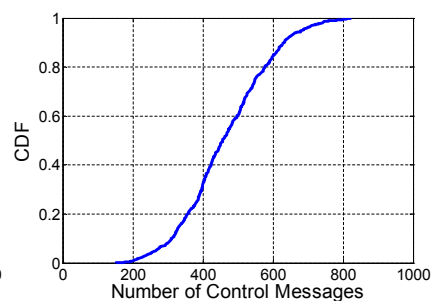

(b) Unstable-connectivity network
Fig. 1: CDF of control messages every node transmits.

control messages. When nodes are in the stable connectivity, the network is stable as well, and few control messages are needed to discover and maintain the routes.

This observation motivates us to use control message transmission rate to design a new routing metric for RPL to discover stable routes, improving the reliability of RPL.

\section{SRPL: STABILITY METRIC BASED RPL}

In this section, we present our stability metric based RPL that leverages control message transmission rate to find stable routes. We also present our passive and lightweight network layer ETX measurement technique for data collection in LLNs.

\section{A. Stability Index}

In RPL, control messages of DIO, DIS and DAO are responsible for constructing and maintaining DODAG topology. Control message transmission rate imply if a node is stable enough to be selected as parent, i.e., next hop node. For instance, forwarding packets to nodes that often transmit DIS messages might not be a wise decision, as DIS transmissions are implying nodes lose connections frequently. Nodes transmitting DIO messages frequently may not be stable as well. They probably have disconnected neighboring nodes and may forward packets to unstable neighboring nodes in the next hop.

To quantify stability, we introduce a new routing metric for RPL called Stability Index (SI). A node can compute two types of SIs, i.e., node SI and DODAG SI. The former is to measure how stable a node is, while the latter is to measure how stable a DODAG topology is. Based on the two types of SIs, a node first selects the most stable DODAG to join and then selects the most stable nodes in the DODAG as its parents. A stable route is the route that is composed of the stable nodes.

To measure the SIs, a hearing window of control messages, $H W_{C}$, is introduced at each node to record the receipt of control messages from the interested neighbors. RPL control messages are generally large in size. For the sake of reducing the memory usage, only the metadata of a control message is recorded rather than entire control message. To calculate node SI, the metadata is \{Sender ID, Message Type, Timestamp\}. For computing DODAG SI, the metadata is RRPInstanceID, DODAGID, DODAGVersionNumber, Sender ID, Message Type, Timestamp $\}. H W_{C}$ works as sliding window. Records are organized in the ascending order of time.

$H W_{C}$ can be size-based or time-based. For the size-based, size (i.e., the maximum number of records) of $H W_{C}$ is fixed.
Records of newly received control messages overwrite old ones if the $H W_{C}$ is full. For the time-based, a node records the received control messages in past time period, no matter how much memory is used. Size-based $H W_{C}$ has advantage in controlling memory usage. However, a burst of control message transmissions by a neighbor may saturate a node's sizebased $H W_{C}$. Time-based $H W_{C}$ records all control messages overheard, and however, may use up a node's memory. In this work, the size-based $H W_{C}$ is used.

We use Fig. 2 to illustrate the computation of the node SI and the DODAG SI. Fig. 2 shows the neighborhood of a node $\mathrm{A}$, in which node $\mathrm{A}$ and its neighbors $\mathrm{B}, \mathrm{C}$ and $\mathrm{D}$ belong to the DODAG- $f$ and the rest of node A's neighbors $\mathrm{A}_{1}, \mathrm{~A}_{2}, \ldots$, $\mathrm{A}_{n}$ belong to the DODAG- $g$.

Node A can measure SIs for all neighboring nodes. For a typical neighbor N, node A measures node N's SI as follows:

$$
S I_{A}^{N}=\frac{w_{d i s} * n_{d i s}^{N}+w_{d i o} * n_{d i o}^{N}+w_{d a o} * n_{d a o}^{N}}{T}
$$

where $w_{d i s}, w_{d i o}$ and $w_{d a o}$ are non-negative weights that account for the importance of DIS, DIO and DAO messages with respect to stability, respectively; $n_{d i s}^{N}, n_{d i o}^{N}$ and $n_{d a o}^{N}$ are the numbers of DIS, DIO and DAO messages transmitted by node $\mathrm{N}$ and overheard by node $\mathrm{A}$ in past $T$ time period, respectively; $T$ is the length of time period used to calculate SI. Node A can also calculate its own SI by using Equation 1. To do so, node A needs to count the numbers of DIS, DIO and DAO messages it transmitted in past $T$ time period.

Node A can measure SI for a neighboring DODAG that has node A and/or at least one of A's neighbors as the member. Typically, node A measures SI of DODAG- $g\left(\mathrm{D}_{g}\right)$ as follows:

$$
S I_{A}^{D_{g}}=\frac{\sum_{i=1}^{n}\left(w_{d i s} * n_{d i s}^{A_{i}}+w_{d i o} * n_{d i o}^{A_{i}}+w_{d a o} * n_{d a o}^{A_{i}}\right)}{n * T}
$$

where $n_{d i s}^{A_{i}}, n_{d i o}^{A_{i}}$ and $n_{d a o}^{A_{i}}$ are the numbers of DIS, DIO and DAO messages transmitted by neighbor $\mathrm{A}_{i}$ and overheard by node A in past $T$ time period $(1 \leq i \leq n)$, respectively; $w_{d i s}$, $w_{d i o}, w_{d a o}$ and $T$ have same meanings as in Equation 1, but not necessarily same values.

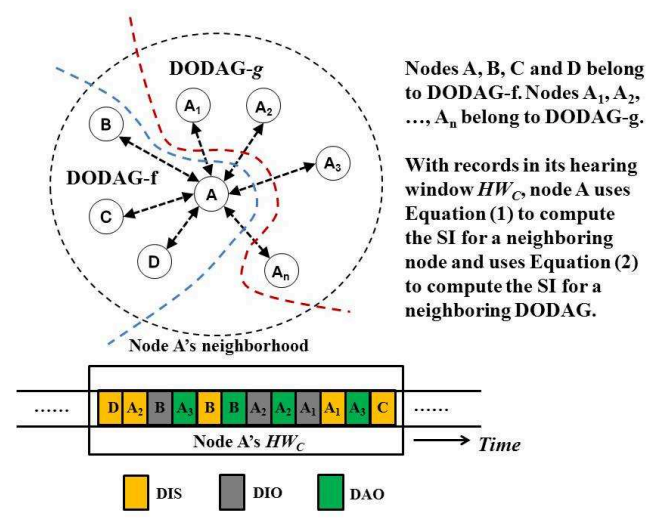

Fig. 2: An example of SI computation.

For the size-based $H W_{C}$, records in $H W_{C}$ may become stale and waste memory. To address this issue, we introduce a 
timer Timer $_{C}$ to periodically delete the records older than $T_{C}-T$ from $H W_{C}$, where $T_{C}$ is the current time. This mechanism is critical for LLN nodes.

There are several advantages in our mechanism of computing SIs. (1) Scalable: SIs are computed by each node independently, and the computation is based on each node's local view of neighboring nodes or DODAGs. (2) Passive: Nodes compute SIs by overhearing the transmissions of control messages only. There is no communication overhead incurred. (3) Independent: Computation of SI is independent from any other protocols or layers. Only information from RPL is used.

\section{B. Passive and Bi-Directional Network Layer ETX}

RFC 6551 [6] defines the ETX metric for RPL as the number of transmissions required to successfully deliver a packet to the destination. But, it does not define any specific formulas. In general, the ETX of a link is defined as:

$$
E T X=\frac{1}{D_{f} * D_{r}},
$$

where $D_{f}$ is the forward delivery rate of the link (e.g., from a child to a parent), and $D_{r}$ is the reversed delivery rate of the link (e.g., from a parent to a child).

The existing network layer ETX measurement methods put pressures on highly limited resources of LLN nodes. We introduce a passive and lightweight method to measure bidirectional ETX at the network layer. The proposed method does not make any assumptions on link layer or physical layer, and does not depend on the hop-to-hop network layer ACK. It only assumes that the data sink identifies missing packets and polls failure nodes for re-transmission. Poll message can be unicasted to an individual node or multicasted to multiple nodes.

Fig. 3 illustrates an example in which nodes transmit data packets to the sink $S$. The solid links (e.g., B to A) indicate the direction of data packet transmission. The dashed links (e.g., B to C) indicate data packet overhearing (e.g., when node $\mathrm{B}$ transmits data packets to node $\mathrm{A}$, node $\mathrm{C}$ overhears the packets although the packets are not destined to node $\mathrm{C}$ ). Sink $S$ polls node $F$ by transmitting poll message because $S$ does not receive the expected data packet from node $F$.

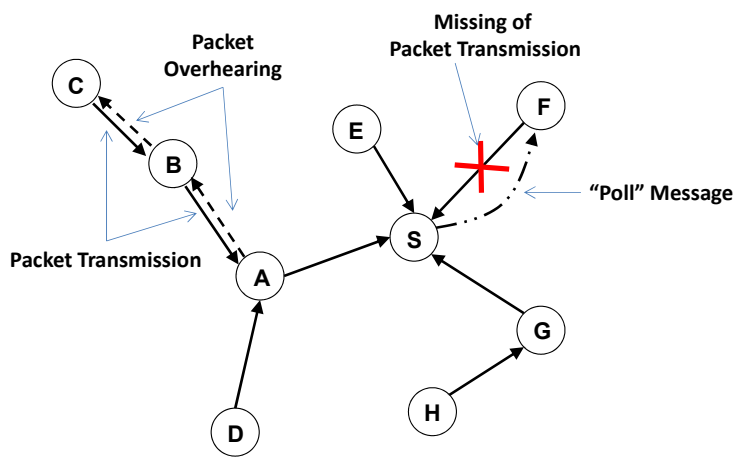

Fig. 3: An example of data collection in LLN.

To measure ETX, a node is only interested in the overheard data packets generated by a parent, not the data packets relayed by the parent (e.g., node B is only interested in data packets of source $A$, not the data packets of source D).
1) Measuring $D_{f}$ : For measuring the $D_{f}$, given a node $Q$, the probability that $Q$ successfully delivers a packet to a data sink $S$ via a path $Q \rightsquigarrow S$ can be measured as:

$$
D_{f}^{\psi_{Q}^{S}}=\frac{N_{Q}^{e x p}-N_{Q}^{p o l l}}{N_{Q}^{e x p}},
$$

where $\psi_{Q}^{S}$ represents the path $Q \rightsquigarrow S, N_{Q}^{e x p}$ is the number of data packets sink $S$ expects to receive from the node $Q$, and $N_{Q}^{p o l t}$ is the number of poll messages node $Q$ receives from sink $S . N_{Q}^{e x p}-N_{Q}^{\text {poll }}$ can be used to estimate the number of data packets successfully delivered.

If node $Q$ has data sink $S$ as its parent via a link $l_{Q}^{S}$, then the Equation 4 can represent the forward delivery rate of $l_{Q}^{S}$ by replacing $\psi_{Q}^{S}$ with $l_{Q}^{S}$.

If the node $Q$ has another node $P$ as its parent via a link $l_{Q}^{P}$, then the forward delivery rate of $l_{Q}^{P}$ can be estimated as:

$$
D_{f}^{l_{Q}^{P}}=\frac{D_{f}^{\psi_{Q}^{S}}}{D_{f}^{\psi_{P}^{S}}},
$$

where $D_{f}^{\psi_{Q}^{S}}, D_{f}^{\psi_{P}^{S}}$ represent the forward delivery rate of paths $\psi_{Q}^{S}$ and $\psi_{P}^{S}$, respectively, and $\psi_{Q}^{S}=l_{Q}^{P} \cup \psi_{P}^{S}$. Node $Q$ obtains $D_{f}^{\psi_{P}^{S}}$ from the ETX Reliability Object of node $P$ 's DIO message, which is transmitted periodically.

To estimate the $N_{Q}^{\text {exp }}$ in the Equation 4, two scenarios of periodic and non-periodic data collection are considered. For the periodic case, nodes periodically send packets to data sink. If the data sink does not receive any packets from a node in last period, it polls that node for re-transmission. Assume nodes send packets at the rate $r$ in past $t$ time period, then $N_{Q}^{e x p}=r * t$. For the non-periodic case, each node adds a sequence number $N_{S E Q}$ in packet, and data sink uses the $N_{S E Q}$ to identify missing packets and polls failure node for re-transmission. We have $N_{Q}^{\text {exp }}=N_{S E Q}^{\text {largest }}-N_{S E Q}^{\text {smallest }}$.

To measure the $N_{Q}^{\text {poll }}$, node $Q$ uses a PollMsgCount to record the timestamps of received poll messages from the data sink in past $t$ time period.

2) Measuring $D_{r}$ : For measuring the $D_{r}$, a node maintains a hearing window of data packet, $H W_{D}$, to record the overheard data packets transmitted by its parents. Specifically, $H W_{D}$ manages records of type $\{$ Parent ID, Timestamp $\}$ and is periodically refreshed.

Given the node $Q$ and its parent node $P$, the measurement of $D_{r}^{l_{Q}^{P}}$ for the link $l_{Q}^{P}$ is:

$$
D_{r}^{l_{Q}^{P}}=\frac{N_{P}^{\text {heard }}}{N_{P}^{e x p}},
$$

where $N_{P}^{\text {heard }}$ is the number of packets transmitted by node $P$ and overheard by node $Q$ in past $t$ time period, and $N_{P}^{e x p}$ is the number of data packets expected to be transmitted by $P$. Calculation of $N_{P}^{e x p}$ is same as above.

In case a node has data sink as its parent, which does not generate any data packets but only control messages, the reversed delivery rate is considered as 1 . 


\section{Weave All Together}

The SI, as a new routing metric for RPL, can be separately used or combined with other metrics. In this section, we present how SI metric and ETX metric are applied together to select the reliable routes for forwarding packets.

Algorithm 1 outlines the sRPL that uses both SI and ETX metrics. Different from RPL, sRPL encloses the initialization of $H W_{C}, H W_{D}$ and PollMsgCount when nodes join or switch to a new DODAG. In addition, timer Timer $_{C}$ is reset periodically for refreshing $H W_{C}, H W_{D}$ and PollM sgCount. The receipt of control messages or data packets is recorded into $H W_{C}$ or $H W_{D}$, respectively. While making routing decisions, the $H W_{C}$ is used for computing SI according to Equation 1, $H W_{D}$ and PollMsgCount are used for computing ETX according to the Equations 4, 5 and 6.

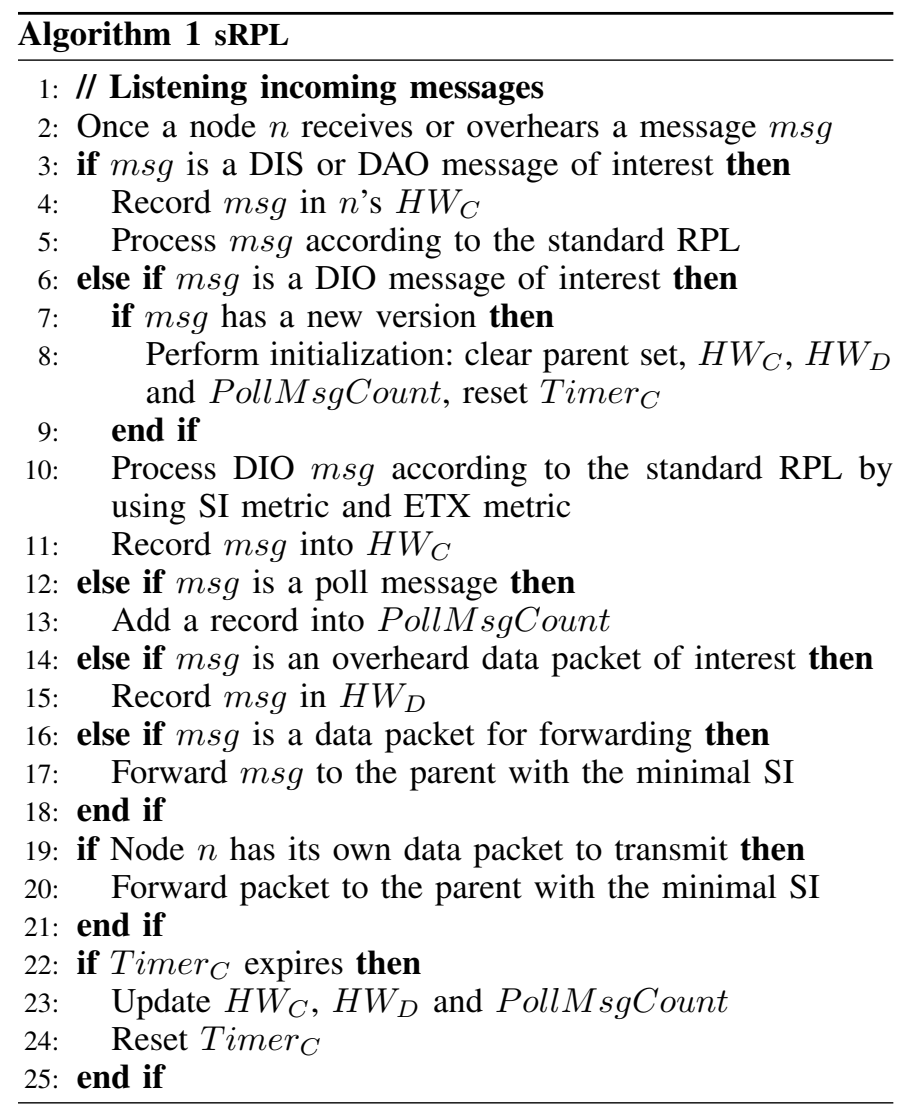

If a node does not have sufficient information to compute SI for a parent, it may apply a default SI value or previous SI value to that parent based on reachability of the parent.

\section{Evaluation}

In this section, we present an extensive evaluation for sRPL using the NS2 simulator. We simulated a wireless LLN consisting of 1024 nodes deployed in a $32 \times 32$ grid. Each unit in the grid represents a $10 \times 10$ square meter real-world field and contains a single node. Each node is randomly placed in its corresponding square field. The IEEE 802.15.4 is adopted as the physical layer and the link layer to simulate an LLN.

We simulated the data collection scenario in which each node transmits packets periodically. More specifically, each node generates packets and sends them to the data sink at the rate of 1 packet per 5 minutes. A data sink is placed at the center of the grid. Due to the space limit, only one data sink is considered in the evaluation. The ratio of weights of $w_{d i s}$, $w_{d i o}$ and $w_{d a o}$ is 1:1:1, i.e., we equally treat control messages of DIS, DIO and DAO in our evaluation. The data collection procedure lasts 24 hours in the simulation. Every result is an average value of ten simulation runs.

To compare the performance of sRPL and the standard RPL, following four cases are simulated: RPL using hop count metric (HC-RPL), RPL using ETX metric (ETX-RPL), RPL using SI metric (SI-RPL), and RPL using combined SI and ETX metrics (SI-ETX-RPL). SI-RPL and SI-ETX-RPL are examples of sRPL because they are stability metric based.

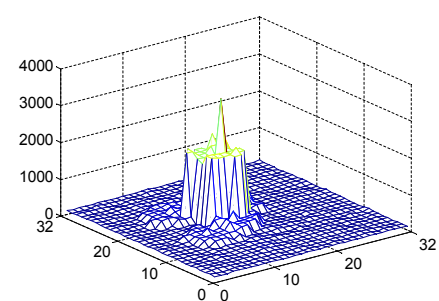

(a) HC-RPL

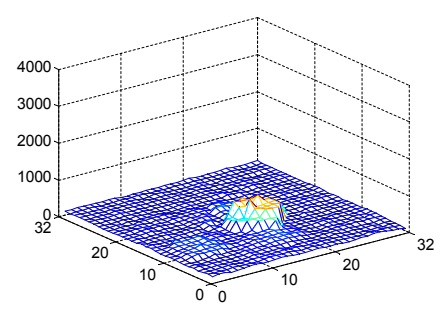

(c) SI-RPL

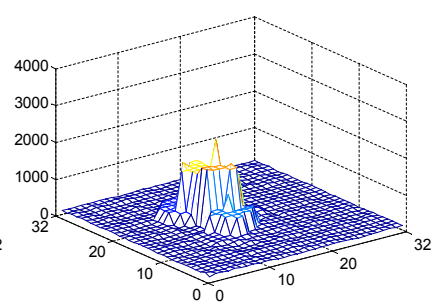

(b) ETX-RPL

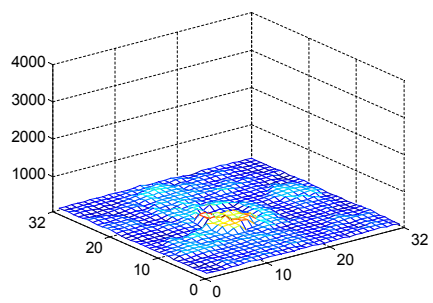

(d) SI-ETX-RPL
Fig. 4: The 3D meshes indicate the number of control messages transmitted by every node.
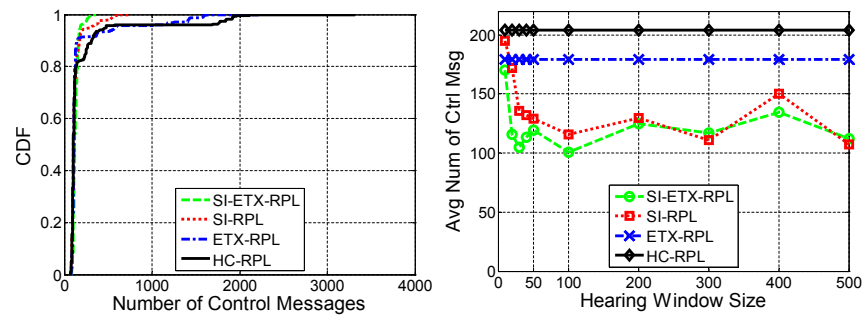

Fig. 5: CDF of control messages ev- Fig. 6: Average number of control ery node transmits

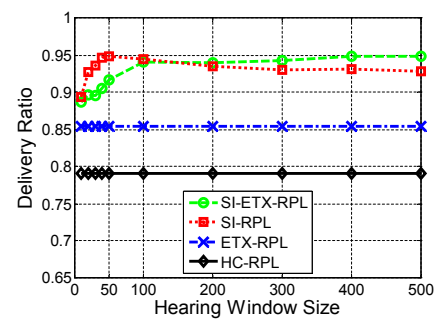

Fig. 7: Packet delivery rate messages

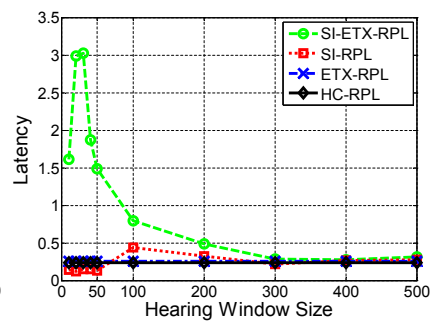

Fig. 8: Average latency 


\section{A. Control Message Overhead}

We collected the number of control messages every node transmitted after the 24-hour simulation, and present them as 3D mesh graphs shown in Fig. 4. As the data sink is placed at the center, the nodes in the central area transmit much more control messages than the others. However, we observed that the spike is reduced significantly when the routing metric is switched from hop count to ETX to SI to combined SI and ETX. It is worth mentioning that the reduction of spikes can balance the power consumption of nodes in entire network. A small number of overloaded nodes can drain their power quickly, and consequently connection holes are generated in the network.

Fig. 5 compares the CDF of the number of control messages transmitted by every node for the four simulation cases. The results validate the 3D mesh graphs. In SI-ETX-RPL, all nodes transmitted less then 400 control messages. In HCRPL, although most nodes transmitted similar number of control messages (less than 400), those close to the data sink transmitted more than 1000 control messages (a few nodes transmit nearly 4000). Fig. 5 shows that sRPL (SI-RPL or SIETX-RPL) has sufficiently reduced the spike by nearly $90 \%$.

Fig. 6 compares the average number of control messages every node transmitted. The results reinforce the improvement that SRPL (SI-ETX-RPL or SI-RPL) can reduce control message overhead by $50 \%$, if compared with HC-RPL. Since HCRPL and ETX-RPL do not involve $H W_{C}$, the corresponding curves are plotted as straight lines.

\section{B. Packet Delivery Rate}

Packet delivery rate directly reflects the reliability of a routing protocol. Fig. 7 illustrates the variation of packet delivery rate with respect to the size of the hearing window $H W_{C}$. We can see that sRPL (SI-ETX-RPL or SI-RPL) outperforms standard RPL (ETX-RPL or HC-RPL). When the size of $H W_{C}$ is small, the packet delivery rates of SIETX-RPL and SI-RPL are slightly better than ETX-RPL, but still much better than HC-RPL. That is because the stability information stored in $H W_{C}$ is insufficient to generate accurate results. The performance of SI-ETX-RPL increases when the size of $H W_{C}$ increase from 10 to 50, where SI-ETX-RPL improves packet delivery rate by $20 \%$ compared with HCRPL. The performance of SI-RPL increases when the size of $H W_{C}$ increase from 10 to 100 . On the other hand, the packet delivery rate of sRPL decrease when the size of $H W_{C}$ continues to increase. The reason is that $T$ was set to 3000 seconds to reflect the fact that stale records in $H W_{C}$ can result in inaccurate routing decisions. These results provide reference base for selecting the size of $H W_{C}$ and the time length $T$.

\section{Latency}

Fig. 8 shows the average latency of packets successfully delivered. HC-RPL and ETX-RPL feature the shortest latency, SI-RPL maintains slightly longer latency, and SI-ETX-RPL exhibits the longest latency. Compared with HC-RPL whose routes prefer shorter paths, SI-ETX-RPL selects more reliable routes with more hops. In addition, as the number of hops increases, the queuing time increases correspondingly. Even though, the latency is still bounded by about $3 \mathrm{~s}$.

\section{CONClusion}

We propose a stability metric based routing protocol called sRPL to discover more stable routes for data collection in LLNs. We introduce a new routing metric of stability index (SI), which uses control message transmission rate to measure the stability of node and routing topology. SI is calculated by passively monitoring control message transmissions with no additional communication overhead. Based on SI, sRPL selects more stable nodes to form more stable routes. In addition, we present a passive and lightweight network layer technique to measure the bi-directional ETX. This technique does require any information from lower layers and only monitors data packet transmissions. As an example of sRPL, SI metric is combined with ETX metric to discover reliable routes for data collection in LLNs. Simulation results show that SRPL improves the performance of RPL in terms of packet delivery rate and control message overhead and maintains similar packet delay. sRPL can reduce the control message overhead by $90 \%$ and improve packet delivery rate by $20 \%$.

\section{REFERENCES}

[1] T. Winter, P. Thuber, B. Brandt, and et al, "RPL: IPv6 Routing Protocol for Low-Power and Lossy Networks," http://tools.ietf.org/html/rfc6550, IETF, 2012.

[2] E. Ancillotti, R. Bruno, and M. Conti, "The Role of the RPL Routing Protocol for Smart Grid Communications," Communications Magazine, IEEE, vol. 51, no. 1, pp. 75-83, 2013.

[3] N. Accettura, L. Grieco, and P. Camarda, "Performance Analysis of the RPL Routing Protocol," in 2011 IEEE International Conference on Mechatronics. IEEE, 2011.

[4] E. Ancillotti, R. Bruno, and M. Conti, "RPL Routing Protocol in Advanced Metering Infrastructures: An Analysis of the Unreliability Problems," in Sustainable Internet and ICT for Sustainability (SustainIT), 2012. IEEE, 2012, pp. 1-10.

[5] M. R. Khan, "Performance and Route Stability Analysis of RPL Protocol," KTH Royal Institute of Technology, 2012.

[6] J. Vasseur, M. Kim, K. Pister, N. Dejean, and D. Barthel, "Routing Metrics Used for Path Calculation in Low-Power and Lossy Networks," http://tools.ietf.org/html/rfc6551, IETF, 2012.

[7] D. S. De Couto, D. Aguayo, J. Bicket, and R. Morris, "A HighThroughput Path Metric for Multi-Hop Wireless Routing," Wireless Networks, vol. 11, no. 4, pp. 419-434, 2005.

[8] S. M. Das, H. Pucha, K. Papagiannaki, and Y. C. Hu, "Studying Wireless Routing Link Metric Dynamics," in Proceedings of the 7th ACM SIGCOMM conference on Internet measurement. ACM, 2007, pp. 327-332.

[9] K.-H. Kim and K. G. Shin, "On Accurate Measurement of Link Quality in Multi-Hop Wireless Mesh Networks," in Proceedings of the 12th annual international conference on Mobile computing and networking. ACM, 2006, pp. 38-49.

[10] D. Wang, Z. Tao, J. Zhang, and A. A. Abouzeid, "RPL Based Routing for Advanced Metering Infrastructure in Smart Grid," in Communications Workshops (ICC), 2010 IEEE International Conference on. IEEE, 2010, pp. 1-6.

[11] S. Dawans, S. Duquennoy, and O. Bonaventure, "On Link Estimation in Dense RPL Deployments," in Proceedings of the 7th IEEE International Workshop on Practical Issues in Building Sensor Network Applications 2012. IEEE, 2012, pp. 956-959.

[12] S. Yoneyama, K. Nakano, and T. Kobayashi, "Wireless Mesh Network Communication Unit for Smart Meters Enabling LowCost and Stable Communication in Smart Grid Systems," http://www.toshiba.co.jp/tech/review/2012/07/67-07pdf/f01.pdf, Toshiba Corporation, 2012.

[13] X. Liu, J. Guo, G. Bhatti, P. Orlik, and K. Parsons, "Load Balanced Routing for Low Power and Lossy Networks," in Wireless Communications and Networking Conference (WCNC), 2013 IEEE. IEEE, 2013. 